\title{
Future evolution in a backreaction model and the analogous scalar field cosmology
}

\author{
Amna Ali, ${ }^{\dagger}$ A. S. Majumdar ${ }^{\dagger}$ \\ $\dagger^{\dagger}$ S. N. Bose National Centre for Basic Sciences, Block JD, Sector-III, Salt Lake, Kolkata \\ 700106, India.
}

\begin{abstract}
We investigate the future evolution of the universe using the Buchert framework for averaged backreaction in the context of a two-domain partition of the universe. We show that this approach allows for the possibility of the global acceleration vanishing at a finite future time, provided that none of the subdomains accelerate individually. The model at large scales is analogously described in terms of a homogeneous scalar field emerging with a potential that is fixed and free from phenomenological parametrization. The dynamics of this scalar field is explored in the analogous FLRW cosmology. We use observational data from Type Ia Supernovae, Baryon Acoustic Oscillations, and Cosmic Microwave Background to constrain the parameters of the model for a viable cosmology, providing the corresponding likelihood contours.
\end{abstract}




\section{Contents}

1 Introduction 1

2 Buchert's backreaction formalism $\quad 3$

3 Two scale partitioning of the Universe and the emerging scalar field 5

4 Evolution of scalar field in global domain $\quad 10$

5 Data Analysis $\quad 12$

6 Conclusion $\quad 16$

$\begin{array}{llr}7 & \text { ACKNOWLEDGEMENTS } & 17\end{array}$

\section{Introduction}

The standard Friedmann-Lemaître-Robertson-Walker (FLRW) cosmological model relies on the Cosmological Principle i.e., the universe is exactly homogeneous and isotropic. The Cosmological principle is however, not a valid assumption at all scales as from the observations of the large scale structure of universe, we know that there exists matter inhomogeneities up to the scales of super-clusters of galaxies. Moreover, the success of FLRW cosmology comes with a cost of assuming that $95 \%$ of energy content of our universe is still unknown and is in form of two dark sectors viz. "dark matter" and "dark energy". Existence of dark matter has been established for a long time. Yet, in spite of numerous ongoing projects there is no direct detection of it till date. The late time cosmic acceleration attributed to dark energy is supported by precise cosmological observations, such as Type Ia supernovae [1], cosmic microwave background anisotropies [2], large scale structure formation [3], baryon oscillations [4], and weak lensing [5]. The theoretical understanding of dark energy is far from satisfactory, even if one accepts the cosmological constant ( $\Lambda$ ) [6] as an additional constant of nature, with the fine tuning and coincidence problem yet to be resolved.

To address these issues a variety of approaches that modify or extend the standard picture have been proposed, such as scalar field models [7-11], and modified gravity models $[12-15]$, to name a few. Most of these approaches are however plagued with problems of their own, primarily to do with naturalness of the considered scenarios. On the other hand, from the observations of large scale structure of the universe a question arises that, can the local matter inhomogeneities be linked to global cosmological components such as dark matter and dark energy? To address this question one needs to adopt an alternative approach to the concordance model and consider the possibility that the dark components could emerge from a suitable averaging procedure applicable to inhomogeneous sources. This approach where the effect of deviations from exact homogeneity and isotropy on the average expansion is studied is called the backreaction approach. Such an approach generalizes the cosmological principle, and considers the universe to be only statistically homogeneous and isotropic at very large scales.

Several averaging techniques [16-21] have been developed to study the relevance of backreaction on the average large scale cosmological dynamics and to describe the late time 
cosmic evolution[22, 23]. One of them is Buchert's approach which relies of an average of scalar quantities over spatial hypersurfaces [24-30]. Based on the framework developed by Buchert it has been shown [26-28] that backreaction from inhomogeneities could lead to an accelerated expansion of the present universe. Application of the Buchert framework could however be responsible for an apparent acceleration due to the difference in lapse of time for the underdense and overdense regions [29]. Extrapolation of the Buchert model further predicts the slowing down of the present acceleration at a future era, with possible avoidance of the future big rip [30]. The impact of backreaction from inhomogeneities provides an interesting platform for investigating the issue of acceleration during the current epoch without invoking modifications to either GR or the standard model of fundamental forces.

In the Buchert's framework the backreaction fluid can be modelled as a scalar field as shown in [31]. In this analogy the effective energy density and an effective pressure corresponding to the scalar field can be obtained from the kinematic backreaction and the averaged curvature term, which appears as additional terms compared with the standard FLRW dynamics. At scales much larger than the scale of inhomogeneities, the dynamics of the the global domain is then analogous to that of a two-fluid universe, one component being matter, the other one the effective scalar field due to backreaction. Scalar field cosmologies [8] have been widely studied in the quest of understanding dark energy, since they provide the scope of a unified framework for explanation of early inflation with dark energy [7] on the one hand, and also a possibility of a unified view of the presently dominant dark sector [10]. In a recent work the backreaction fluid has been constructed considering a bulk viscous fluid which gives a unified description of dark matter and dark energy [32].

In this paper we investigate the backreaction fluid to see whether it can act as a source of dark energy thereby giving rise to a model analogous to scalar field cosmologies in the standard FRW model. We see a number of advantages in such description. First, the effective scalar field has a well justified status in this theory, and we do not have to consider it as an additional source arising from an extraneous field theory. Second, the inhomogeneities encoded in the backreaction term fix the potential, thereby determining the evolution of the scalar field in it, and therefore, the model does not suffer from phenomenological parametrization. Third, the correspondence allows a realistic reinterpretation of phenomenological models involving scalar fields, as a large variety of potentials are allowed in such models and there is no way in which one can restrict these potentials. By making backreaction responsible for the late time cosmic acceleration the scalar field models can be reinvestigated using a fixed potential. Fourth, the cosmological constant can arise as a particular solution of the averaged background without being present in the Einstein's equations.

In the present work we construct a scalar field model due to backreaction, which is analogous to the quintessence field model of dark energy. Our focus here is to study the future evolution of the presently observed accelerating universe. In order to do so, we first determine the potential of the effective quintessence field in a two scale model of inhomogeneities within the Buchert framework. We then study the evolution of the effective scalar field in the context of the standard FRW background at scales where cosmological principle is assumed to hold true. We perform observational analysis on the model and see how the parameters of the model are constrained.

The plan of the paper is as follows. In the next section we present a brief overview of Buchert's formalism of backreaction involving partitioning of the global domain into overdense and underdense regions. In Section III we present our two scale model with the emergent scalar field. The dynamics of the effective scalar field are presented in Section IV. In 
Section $\mathrm{V}$ we perform observational analysis of our model by considering the data from three different experiments, viz., Type Ia Supernovae, Baryon Acoustic Oscillations, and Cosmic Microwave Background. We conclude with a brief summary of our results in Section VI.

\section{Buchert's backreaction formalism}

Assuming the universe to be filled with an irrotational fluid of dust, the space-time is foliated into flow-orthogonal hypersurfaces with an inhomogeneous 3-metric $g_{i j}$ and the line-element

$$
d s^{2}=-d t^{2}+g_{i j} d X^{i} d X^{j}
$$

where $t$ is the proper time of the hypersurfaces and $X^{i}$ are the spatial coordinates in the hyper-surfaces of constant $t$. This framework is applicable to perfect fluid matter models. The averaged equations for volume expansion and volume acceleration for a compact domain $\mathcal{D}$ from this approach are those found by averaging the Hamiltonian, Raychaudhuri and conservation equations[18, 24-27], and are given by

$$
\begin{gathered}
3 \frac{\ddot{a}_{\mathcal{D}}}{a_{\mathcal{D}}}=-4 \pi G\langle\rho\rangle_{\mathcal{D}}+\mathcal{Q}_{\mathcal{D}}+\Lambda, \\
3 H_{\mathcal{D}}^{2}=8 \pi G\langle\rho\rangle_{\mathcal{D}}-\frac{1}{2}\langle\mathcal{R}\rangle_{\mathcal{D}}-\frac{1}{2} \mathcal{Q}_{\mathcal{D}}+\Lambda, \\
\langle\dot{\rho}\rangle_{\mathcal{D}}+3 H_{\mathcal{D}}\langle\rho\rangle_{\mathcal{D}}=0
\end{gathered}
$$

where $a_{\mathcal{D}}$ is the volume scale factor of the global domain $\mathcal{D},\langle\rho\rangle_{\mathcal{D}}$ is the average matter density of the domain, $\langle\mathcal{R}\rangle_{\mathcal{D}}$ is the is the averaged three curvature $\left\langle{ }^{3} R\right\rangle_{\mathcal{D}}$, and $H_{\mathcal{D}}$ is the Hubble rate. The angular brackets denote a volume average throughout the region $\mathcal{D}$, and $\mathcal{Q}_{\mathcal{D}}$ is the the backreaction term that quantifies the averaged effect of inhomogeneities in the background. It is defined as

$$
\mathcal{Q}_{\mathcal{D}}=\frac{2}{3}\left(\left\langle\theta^{2}\right\rangle_{\mathcal{D}}-\langle\theta\rangle_{\mathcal{D}}^{2}\right)-2\left\langle\sigma^{2}\right\rangle_{\mathcal{D}}
$$

where $\theta$ is the volume expansion rate and $\sigma^{2}=1 / 2 \sigma_{i j} \sigma^{i j}$ is the squared rate of shear. The volume average of a scalar quantity $\langle f\rangle_{\mathcal{D}}(t)$ over the rest mass preserving domain $\mathcal{D}$, is given by

$$
\langle f\rangle_{\mathcal{D}}(t)=\frac{1}{|\mathcal{D}|_{g}} \int_{\mathcal{D}} f d \mu_{g},
$$

where $|\mathcal{D}|_{g}=\int_{\mathcal{D}} d \mu_{g}$ is the volume of the domain and $d \mu_{g}=\sqrt{{ }^{(3)} g\left(t, X^{1}, X^{2}, X^{3}\right)} d X^{1} d X^{2} d X^{3}$. The volume scale factor $a_{\mathcal{D}}$ is defined as:

$$
a_{\mathcal{D}}(t)=\left(\frac{|\mathcal{D}|_{g}}{\left|\mathcal{D}_{0}\right|_{g}}\right)^{1 / 3}
$$

where $\left|\mathcal{D}_{0}\right|_{g}$ is the volume of the domain at a reference time $t_{0}$ which we may take as the present time. The quantities $\mathcal{Q}_{\mathcal{D}}$ and $\langle\mathcal{R}\rangle_{\mathcal{D}}$ obey the consistency relation

$$
\frac{1}{a_{\mathcal{D}}^{6}} \partial_{t}\left(a_{\mathcal{D}}^{6} \mathcal{Q}_{\mathcal{D}}\right)+\frac{1}{a_{\mathcal{D}}^{2}} \partial_{t}\left(a_{\mathcal{D}}^{2}\langle\mathcal{R}\rangle_{\mathcal{D}}\right)=0,
$$


which connects (2.2) and (2.3), assuming that the expansion equation as integral of the acceleration equation. While the mass conservation law for matter is sufficient for the case of a smooth universe, the additional equation (2.8) connects the averaged intrinsic and extrinsic curvature invariants when the effect of inhomogeneities on the background metric are taken into consideration. The new second conservation equation (2.8) describes an interaction between structure formation and the background curvature. The dynamical coupling of $\mathcal{Q}_{\mathcal{D}}$ to the average scalar curvature $\langle\mathcal{R}\rangle_{\mathcal{D}}$ implies that the temporal behaviour of the averaged curvature is different from that of the standard FRW constant curvature model where it is restricted to an $a_{\mathcal{D}}^{-2}$ behaviour, whereas in the present case it is more dynamical since it can be any function of $a_{\mathcal{D}}$.

At scales much larger than the scale of inhomogeneites, the global domain is assumed to be homogeneous, and the above equations can formally be recast into standard Friedmann equations for an effective perfect fluid energy momentum tensor with new effective sources as [31]:

$$
\begin{array}{r}
\rho_{\text {eff }}^{\mathcal{D}}=\langle\rho\rangle_{\mathcal{D}}-\frac{1}{16 \pi G} \mathcal{Q}_{\mathcal{D}}-\frac{1}{16 \pi G}\langle\mathcal{R}\rangle_{\mathcal{D}} \\
P_{\text {eff }}^{\mathcal{D}}=-\frac{1}{16 \pi G} \mathcal{Q}_{\mathcal{D}}+\frac{1}{16 \pi G}\langle\mathcal{R}\rangle_{\mathcal{D}}
\end{array}
$$

such that

$$
\begin{gathered}
3 \frac{\ddot{a}_{\mathcal{D}}}{a_{\mathcal{D}}}=-4 \pi G\left(\rho_{\text {eff }}^{\mathcal{D}}+3 P_{\text {eff }}^{\mathcal{D}}\right)+\Lambda, \\
3 H_{\mathcal{D}}^{2}=8 \pi G \rho_{\text {eff }}^{\mathcal{D}}+\Lambda, \\
\dot{\rho}_{\text {eff }}^{\mathcal{D}}+3 H_{\mathcal{D}}\left(\rho_{\text {eff }}^{\mathcal{D}}+P_{\text {eff }}^{\mathcal{D}}\right)=0 .
\end{gathered}
$$

Eqs.(2.10), (2.11) and (2.12) correspond to the eqs.(2.2), (2.3) and (2.4) respectively.

Since the scalar functions are domain dependent, following [26] the homogeneous global domain is partitioned into noninteracting subregions $\mathcal{F}_{\ell}$ which themselves consist of elementary space entities $\mathcal{F}_{\ell}^{(\alpha)}$ that is associated with some averaging length scale. Mathematically $\mathcal{D}=\cup_{\ell} \mathcal{F}_{\ell}$, where $\mathcal{F}_{\ell}=\cup_{\alpha} \mathcal{F}_{\ell}^{(\alpha)}$ and $\mathcal{F}_{\ell}^{(\alpha)} \cap \mathcal{F}_{m}^{(\beta)}=\emptyset$ for all $\alpha \neq \beta$ and $\ell \neq m$. One can construct the average of the scalar function $f$ on the domain $\mathcal{D}$ as in eqn.(2.2) summing all the averages $f$ on the subregions $\mathcal{F}_{\ell}$ as,

$$
\langle f\rangle_{\mathcal{D}}=\sum_{\ell}|\mathcal{D}|_{g}^{-1} \sum_{\alpha} \int_{\mathcal{F}_{\ell}^{(\alpha)}} f d \mu_{g}=\sum_{\ell} \lambda_{\ell}\langle f\rangle_{\mathcal{F}_{\ell}}
$$

where $\lambda_{\ell}=\left|\mathcal{F}_{\ell}\right|_{g} /|\mathcal{D}|_{g}$, is the volume fraction of the subregion $\mathcal{F}_{\ell}$. The scalar quantities $\langle\rho\rangle_{\mathcal{D}}$, $<\mathcal{R}>_{\mathcal{D}}$ and $H_{\mathcal{D}}$ follow the above equation except $\mathcal{Q}_{\mathcal{D}}$, since from its definition in eqn.(2.3) we notice that due to the term $\langle\theta\rangle_{\mathcal{D}}^{2}$ it does not split in a simple manner. Instead, the correct formula turns out to be

$$
\mathcal{Q}_{\mathcal{D}}=\sum_{\mathcal{D}} \lambda_{\ell} \mathcal{Q}_{\ell}+3 \sum_{\ell \neq m} \lambda_{\ell} \lambda_{m}\left(H_{\ell}-H_{m}\right)^{2}
$$

where $\mathcal{Q}_{\ell}$ and $H_{\ell}$ are defined in the subregion $\mathcal{F}_{\ell}$ in the same way as $\mathcal{Q}_{\mathcal{D}}$ and $H_{\mathcal{D}}$ are defined in $\mathcal{D}$. The shear part $\left\langle\sigma^{2}\right\rangle_{\mathcal{F}_{\ell}}$ is completely absorbed in $\mathcal{Q}_{\ell}$, whereas the variance of the local expansion rates $\left\langle\theta^{2}\right\rangle_{\mathcal{D}}-\langle\theta\rangle_{\mathcal{D}}^{2}$ is partly contained in $\mathcal{Q}_{\ell}$ and partly generates the extra term 
$3 \sum_{\ell \neq m} \lambda_{\ell} \lambda_{m}\left(H_{\ell}-H_{m}\right)^{2}$. This is because the variance $\left\langle\theta^{2}\right\rangle_{\mathcal{F}_{\ell}}-\langle\theta\rangle_{\mathcal{F}_{\ell}}^{2}$ that is present in the backreaction term $\mathcal{Q}_{\ell}$ of a subregion takes care only of the points inside the subregion $\mathcal{F}_{\ell}$. To take into account of all the variance that comes from combining points of the subregion $\mathcal{F}_{\ell}$ with others in $\mathcal{F}_{m}$, the extra term which contains the averaged Hubble rate in above equation emerges. It is to be noted here that this formulation of the backreaction only holds in the case when there is no overlap between the different subregions.

As the volume of the global domain $\mathcal{D}$ is equal to the sum of volumes of all individual sub domains such that $|\mathcal{D}|_{g}=\sum_{\ell}\left|\mathcal{F}_{\ell}\right|_{g}$, the corresponding scale-factor $a_{\ell}$ for each of the subregions $\mathcal{F}_{\ell}$ can be defined as

$$
a_{\mathcal{D}}^{3}=\sum_{\ell} \lambda_{\ell_{i}} a_{\ell}^{3}
$$

where $\lambda_{\ell_{i}}=\left|\mathcal{F}_{\ell_{i}}\right|_{g} /\left|\mathcal{D}_{i}\right|_{g}$ is the initial volume fraction of the subregion $\mathcal{F}_{\ell}$. If we now twice differentiate this equation with respect to the foliation time and use the result for $\dot{a}_{\ell}$ from (2.3), we then get the expression that relates the acceleration of the global domain to that of the subdomains:

$$
\frac{\ddot{a}_{\mathcal{D}}}{a_{\mathcal{D}}}=\sum_{\ell} \lambda_{\ell} \frac{\ddot{a}_{\ell}(t)}{a_{\ell}(t)}+\sum_{\ell \neq m} \lambda_{\ell} \lambda_{m}\left(H_{\ell}-H_{m}\right)^{2} .
$$

From the above equation we notice that even if the subdomains decelerate individually, the second term in the equation may counterbalance the first one to lead to global accelerated expansion.

\section{Two scale partitioning of the Universe and the emerging scalar field}

For our purpose we consider the universe to be partitioned into two subdomains: (i) all overdense regions are clubbed together into the domain of over density called wall $\mathcal{M}$, and (ii) all underdense regions are clubbed together into the domain of under density called void $\mathcal{E}$, such that $\mathcal{D}=\mathcal{M} \cup \mathcal{E}$. Now, from the Buchert's formalism as explained in the previous section one obtains $H_{\mathcal{D}}=\lambda_{\mathcal{M}} H_{\mathcal{M}}+\lambda_{\mathcal{E}} H_{\mathcal{E}}$, with similar expressions for $\langle\rho\rangle_{\mathcal{D}}$ and $\langle\mathcal{R}\rangle_{\mathcal{D}}$. Simplifying eqn.(2.5) for the two domain case considered here, we get

$$
\frac{\ddot{a}_{\mathcal{D}}}{a_{\mathcal{D}}}=\lambda_{\mathcal{M}} \frac{\ddot{a}_{\mathcal{M}}}{a_{\mathcal{M}}}+\lambda_{\mathcal{E}} \frac{\ddot{a}_{\mathcal{E}}}{a_{\mathcal{E}}}+2 \lambda_{\mathcal{M}} \lambda_{\mathcal{E}}\left(H_{\mathcal{M}}-H_{\mathcal{E}}\right)^{2}
$$

where $\lambda_{\mathcal{M}}=|\mathcal{M}| /|\mathcal{D}|$ and $\lambda_{\mathcal{E}}=|\mathcal{E}| /|\mathcal{D}|$ are the volume fractions of wall and void respectively. Since $\sum_{\ell} \lambda_{\ell}=1$, therefore,

$$
\lambda_{\mathcal{M}}+\lambda_{\mathcal{E}}=1 .
$$

We assume different behaviours for the scale factors of the two sub regions. The wall $\mu$ is considered to be a closed region with a positive curvature and a deceleration parameter $q_{\mathcal{M}}=-\ddot{a}_{\mathcal{M}} / a_{\mathcal{M}} H_{\mathcal{M}}^{2}>.5$. The scale factor $a_{\mathcal{M}}$ in terms of the time $t$ parametrized by the angle $\theta$ is given by:

$$
\begin{gathered}
a_{\mathcal{M}}=\frac{q_{\mathcal{M}}}{2 q_{\mathcal{M}}-1}(1-\operatorname{Cos}(\theta)), \\
t=\frac{q_{\mathcal{M}}}{2 q_{\mathcal{M}}-1}(\theta-\operatorname{Sin}(\theta)),
\end{gathered}
$$

with $\theta$ varying from 0 to $2 \pi$. The void is considered to be a flat (zero intrinsic curvature domain) with its scale factor $a_{\mathcal{E}}$ assumed to follow a power law:

$$
a_{\mathcal{E}}=c_{\mathcal{E}} t^{\beta},
$$


where $\beta$ and $c_{\mathcal{E}}$ are constants. From eqn.(2.6) the acceleration equation of the global domain is given by

$$
\frac{\ddot{a}_{\mathcal{D}}}{a_{\mathcal{D}}}=\lambda_{\mathcal{M}}\left(-q_{\mathcal{M}} H_{\mathcal{M}}^{2}\right)+\lambda_{\mathcal{E}} \frac{\beta(\beta-1)}{t^{2}}+2 \lambda_{\mathcal{M}} \lambda_{\mathcal{E}}\left(H_{\mathcal{M}}-\frac{\beta}{t}\right)^{2} \text {. }
$$

Since the volume fractions $\lambda_{\mathcal{M}}$ and $\lambda_{\mathcal{E}}$ are constrained by eqn.(3.2), determination of one fixes the other. The volume fraction of the void subdomain $\mathcal{E}$ is given by $\lambda_{\mathcal{E}}=\frac{|\mathcal{E}|}{\mid \mathcal{D}}$, which can be rewritten in terms of the corresponding scale factor as $\lambda_{\mathcal{E}}=\frac{a_{\mathcal{E}}^{3}|\mathcal{E}|}{a_{\mathcal{D}}^{3}|\mathcal{D}|}$, which in turn gives us:

$$
\lambda_{\mathcal{E}}=k \frac{t^{3 \beta}}{a_{\mathcal{D}}^{3}},
$$

where where $k=\frac{\lambda_{\mathcal{E}_{0}} a_{\mathcal{D}_{0}}^{3}}{t_{0}^{3 \beta}}$ is a constant and $\lambda_{\mathcal{E}_{0}}, a_{\mathcal{D}_{0}}, t_{0}$ are the present volume fraction of void region, the present global scale factor and the present time, respectively. An N-body simulation of large scale structure formation [26] indicates the value $\lambda_{\mathcal{M}_{0}}=.09$, and hence using eqn.(3.2), we choose $\lambda_{\mathcal{E}_{0}}=1-\lambda_{\mathcal{M}_{0}}=.91$. Eqn.(3.6) can thus be written as:

$$
\begin{aligned}
\frac{\ddot{a}_{\mathcal{D}}}{a_{\mathcal{D}}}= & \left(1-\frac{k t^{3 \beta}}{a_{\mathcal{D}}^{3}}\right)\left(-q_{\mathcal{M}} H_{\mathcal{M}}^{2}\right)+\frac{k t^{3 \beta}}{a_{\mathcal{D}}^{3}} \frac{\beta(\beta-1)}{t^{2}} \\
& +2 \frac{k t^{3 \beta}}{a_{\mathcal{D}}^{3}}\left(1-\frac{k t^{3 \beta}}{a_{\mathcal{D}}^{3}}\right)\left(H_{\mathcal{M}}-\frac{\beta}{t}\right)^{2} .
\end{aligned}
$$

Now, using eq.(2.9), the potential $V_{\mathcal{D}}\left(\phi_{\mathcal{D}}\right)$ of the effective scalar field $\phi_{\mathcal{D}}$ may be obtained as follows [31]:

$$
\rho_{\text {eff }}^{\mathcal{D}}=\langle\rho\rangle_{\mathcal{D}}+\rho_{\phi}^{\mathcal{D}} ; \quad P_{\text {eff }}^{\mathcal{D}}=P_{\phi}^{\mathcal{D}}
$$

where,

$$
\rho_{\phi}^{\mathcal{D}}=\frac{1}{2} \dot{\phi}_{\mathcal{D}}^{2}+V_{\mathcal{D}} ; \quad P_{\phi}^{\mathcal{D}}=\frac{1}{2} \dot{\phi}_{\mathcal{D}}^{2}-V_{\mathcal{D}}
$$

Setting $\Lambda=0$ in the Eqs.(2.10) and Eqs.(2.11), and using eqs.(2.12),(3.9) and (3.10), we get the potential of the field to be

$$
V_{\mathcal{D}}=\frac{1}{8 \pi G} \frac{\ddot{a}_{\mathcal{D}}}{a_{\mathcal{D}}}+2\left(\frac{\dot{a}_{\mathcal{D}}(t)}{a_{\mathcal{D}}(t)}\right)^{2}-\frac{3}{16 \pi G} \frac{\Omega_{m 0} H_{0}^{2}}{a_{\mathcal{D}}^{3}},
$$

where $\Omega_{m 0}=\rho_{m 0} / 3 H_{0}^{2} M_{p}^{2}, M_{p}=1 / \sqrt{8 \pi G}$. Next, let us define the quantities:

$$
H_{0} t \rightarrow t_{n}, \frac{\phi_{\mathcal{D}}}{M_{p}} \rightarrow \phi, \frac{V_{\mathcal{D}}}{H_{0}^{2} M_{p}^{2}} \rightarrow V
$$

In terms of the above dimensional quantities, the potential becomes:

$$
V(\phi(t))=\frac{\ddot{a}_{\mathcal{D}}(t)}{a_{\mathcal{D}}(t)}+2\left(\frac{\dot{a}_{\mathcal{D}}(t)}{a_{\mathcal{D}}(t)}\right)^{2}-\frac{3 \Omega_{m 0}}{2 a_{\mathcal{D}}(t)^{3}}
$$

From Eqs.2.9 we obtain the following correspondence:

$$
-\frac{1}{8 \pi G} \mathcal{Q}_{\mathcal{D}}=\dot{\phi}(t)-V(\phi) ;-\frac{1}{8 \pi G}\langle\mathcal{R}\rangle_{\mathcal{D}}=3 V(t),
$$




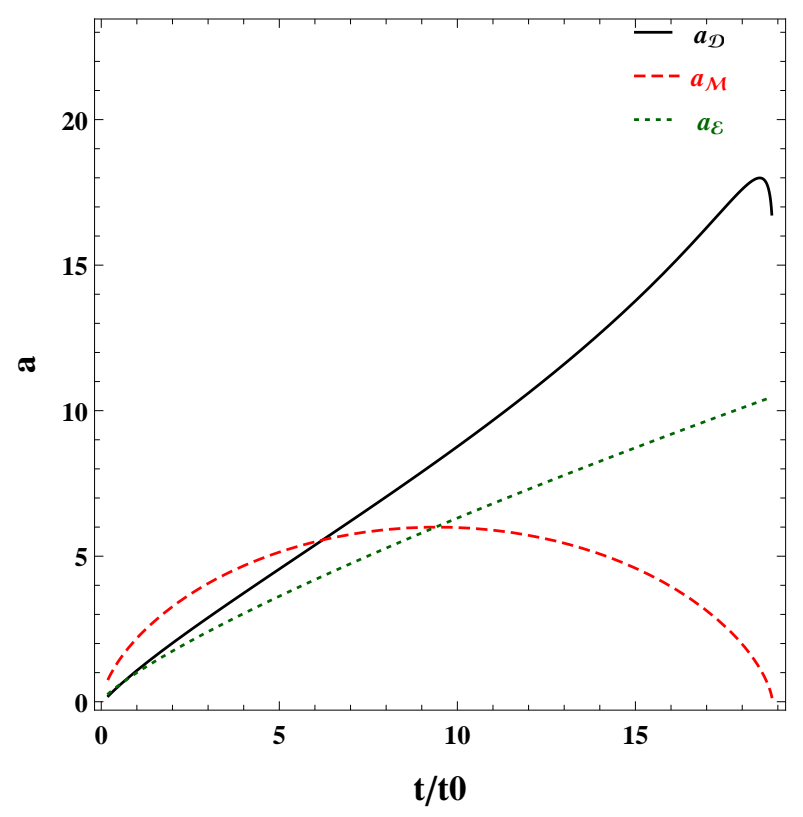

Figure 1: The scale factor of the over-dense subdomain $a_{\mathcal{M}}$ (wall), the under-dense subdomain $a_{\mathcal{E}}$ (void) and the global domain $a_{\mathcal{D}}$ with time(in units of $t / t_{0}$ with $t_{0}$ being the present time) for $q_{\mathcal{M}}=.6$ and $\beta=.7$. The global scale factor $a_{\mathcal{D}}$ first increases giving a period of acceleration due to the addition of two scale factor $\left(a_{\mathcal{M}}, a_{\mathcal{E}}\right)$, and then the collapse in $\mathcal{M}$-region forces the decline of $a_{\mathcal{D}}$ at late times.

Inserting the above equations into the integrability condition 2.8 implies that $\phi(t)$, for $\dot{\phi}(t) \neq$ 0, obeys the Klein-Gordon equation:

$$
\ddot{\phi}(t)+3 H_{\mathcal{D}} \dot{\phi}(t)+\frac{d V(\phi)}{d \phi}=0,
$$

where $t$ is the dimensionless time $\left(t_{n}\right)$ defined in (3.12) (we henceforth drop the subscript for simplicity).

One may now study the consequence of backreaction due to inhomogeneities in terms of the formally equivalent dynamics of a homogeneous, minimally coupled scalar field. Given this correspondence, we can reconstruct the potential in which the field evolves by solving the two equations (3.8) and (3.15) numerically given the initial conditions. For this we chose the initial conditions such that at $t / t_{0} \rightarrow 1$ the present global scale factor $a_{\mathcal{D}_{0}}=1$. Fig.1 shows the evolution of scale factors of the over-dense subdomain $a_{\mathcal{M}}$ (wall), the under-dense subdomain $a_{\mathcal{E}}$ (void) and the global domain $a_{\mathcal{D}}$ respectively, with time. We notice that the scale factor of global domain increases for certain period of time and then decrease in the late future.

We start with a two scale universe where the initial volume fraction of the wall and the void are equal. With time the void expands whereas the wall contracts. Note that even if finally the wall occupies a tiny volume of the global volume, its contraction impacts the evolution of the global scale factor as a result of which the global Hubble parameter changes its sign. The global Hubble parameter is given by $H_{\mathcal{D}}=\lambda_{\mathcal{M}} H_{\mathcal{M}}+\lambda_{\mathcal{E}} H_{\mathcal{E}}$. As time increases, $H_{\mathcal{E}}$ (as per our chosen ansatz given by Eq.(3.5) falls off rapidly compared to $H_{\mathcal{M}}$ (defined by Eqs.(3.3) and (3.4)). Thus, the contribution of the decreasing $\lambda_{\mathcal{M}}$ is compensated by the 


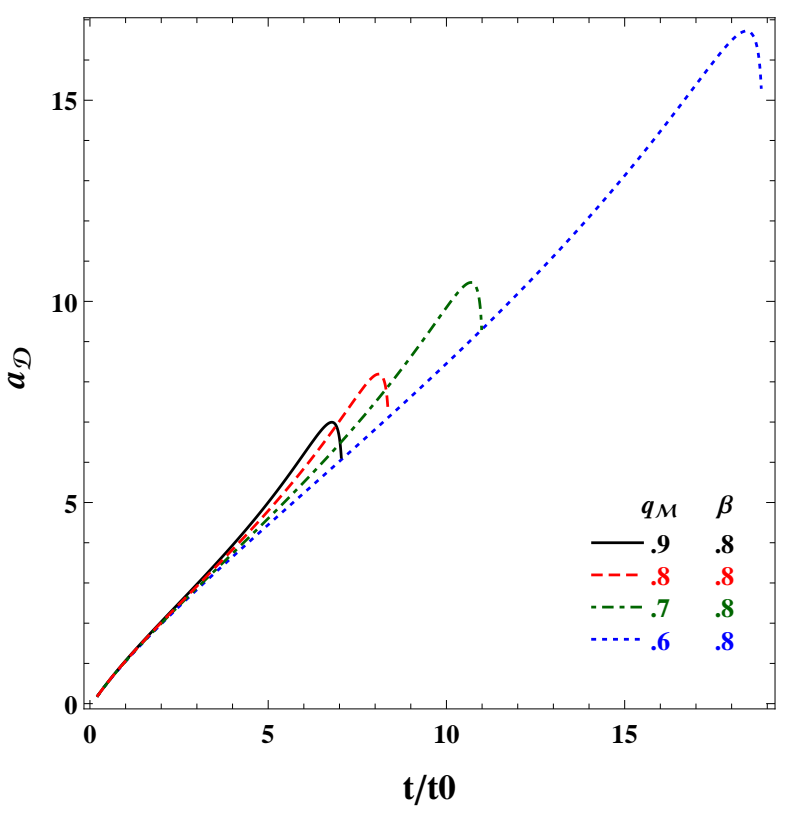

(a)

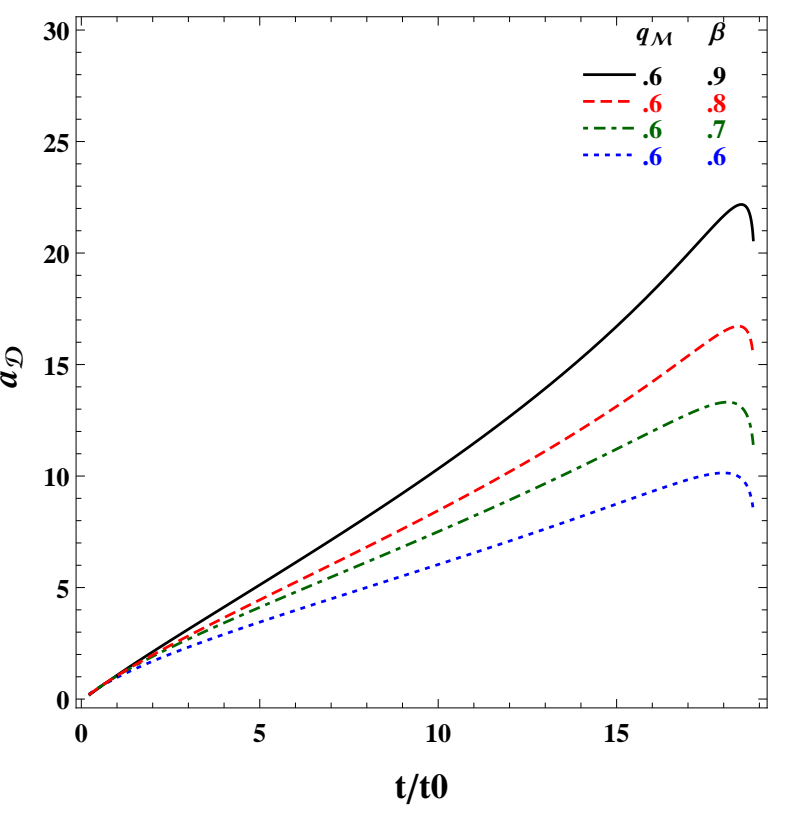

(b)

Figure 2: The left panel (a): the evolution of the scale factor of the global domain $a_{\mathcal{D}}$ with time is shown for different values of $q_{\mathcal{M}}$ keeping $\beta$ fixed. The right panel (b): the evolution of the scale factor of the global domain $a_{\mathcal{D}}$ with time is shown for different values of $\beta$ keeping $q_{\mathcal{M}}$ fixed.

comparative behaviours of $H_{\mathcal{M}}$ and $H_{\mathcal{E}}$ to lead to a net change of sign in the global Hubble parameter at late times. We would however like to add here that the framework adopted for our study may not be adequate for investigating the collapsing phase of the global domain in the far future. Our assumption of the disjoint nature of the wall and void is likely to be invalidated during the collapsing phase in future, as during this the contracting global volume may entail the wall region overlapping with the void. From Fig.2 it is evident that time which marks the decrease of $a_{\mathcal{D}}$ depends on the value of $q_{\mathcal{M}}$, as it determines the time up to which $a_{\mathcal{M}}$ expands. $\beta$ on the other hand determines how much $a_{\mathcal{D}}$ increases before it starts decreasing. We see that $a_{\mathcal{D}}$ increases with increasing $\beta$.

It is interesting to see what happens to the evolution of the universe once the present stage of acceleration sets in. For this we scale the present value of acceleration parameter $\frac{\ddot{a}_{\mathcal{D}}}{a_{\mathcal{D}} H_{\mathcal{D}}^{2}}\left(=-q_{\mathcal{D}}\right)$ to the observed value $(0.5)$ and determine the future evolution of the universe. The global acceleration for fixed $q_{\mathcal{M}}$ and fixed $\beta$ has been plotted in Fig.3. We see that the acceleration reaches maximum at present and then it gradually decreases until it becomes negative. This predicts that the present accelerating phase is succeeded by a future decelerating phase. Next, we study the nature of the potential of the emerging scalar field due to the effect of backreaction. In Fig.4 we plot the dimensionless potential $V$ as a function of field $\phi$. The nature of potential is unique for the parameter $\left(q_{\mathcal{M}}, \beta\right)$, and it is free from other parametrization. Further, in the next section we investigate the dynamics of the emerging scalar field in this potential. 


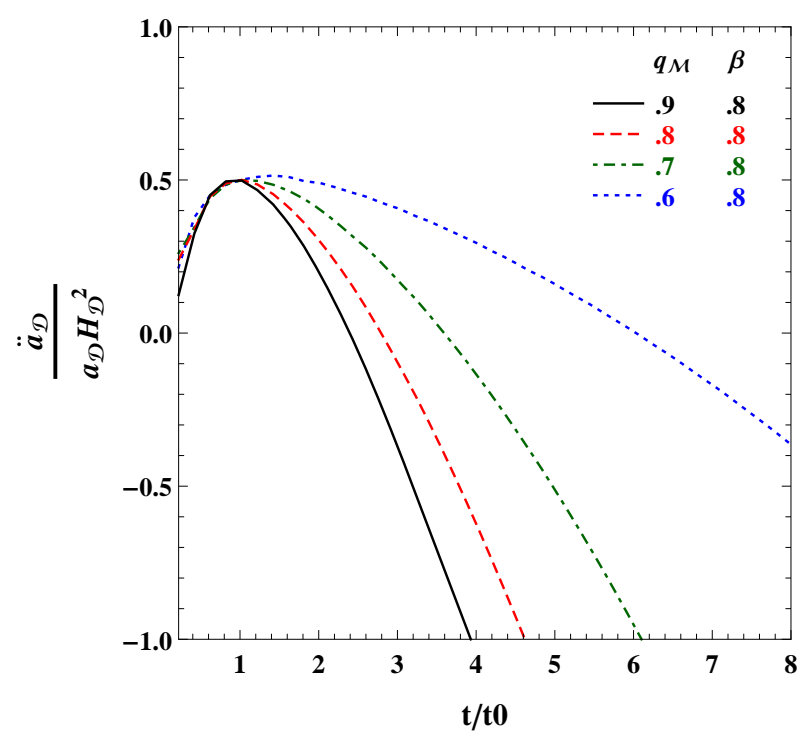

(a)

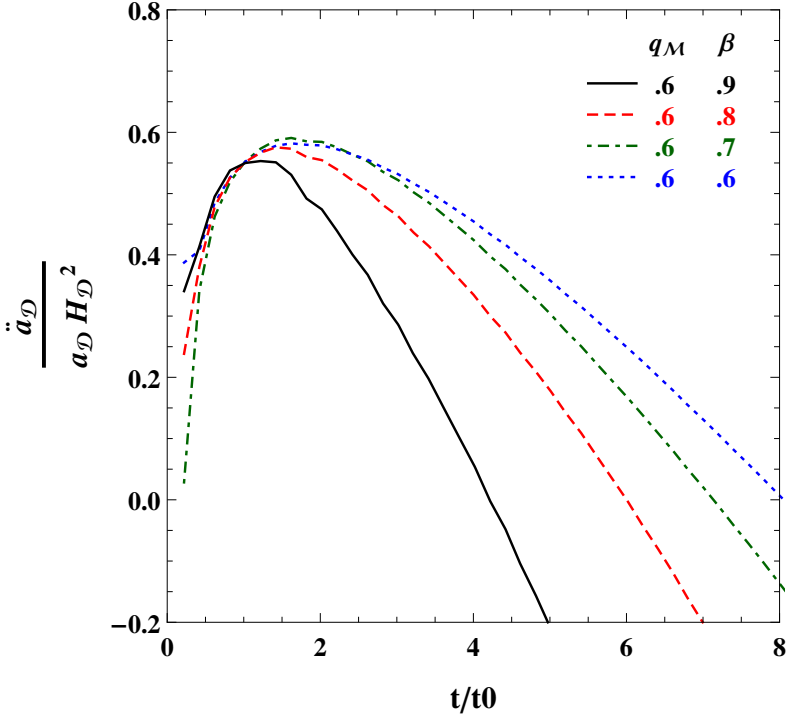

(b)

Figure 3: The left panel (a): the dimensionless global acceleration parameter $\frac{\ddot{a}_{\mathcal{D}}}{a_{\mathcal{D}} H_{\mathcal{D}}^{2}}$ versus time $\left(t / t_{0}\right)$ is shown for different values of $q_{\mathcal{M}}$ keeping $\beta$ fixed. The right panel (b): the dimensionless global acceleration parameter $\frac{\ddot{a}_{\mathcal{D}}}{a_{\mathcal{D}} H_{\mathcal{D}}^{2}}$ vs. time $\left(t / t_{0}\right)$ is shown for different values of $\beta$ keeping $q_{\mathcal{M}}$ fixed.

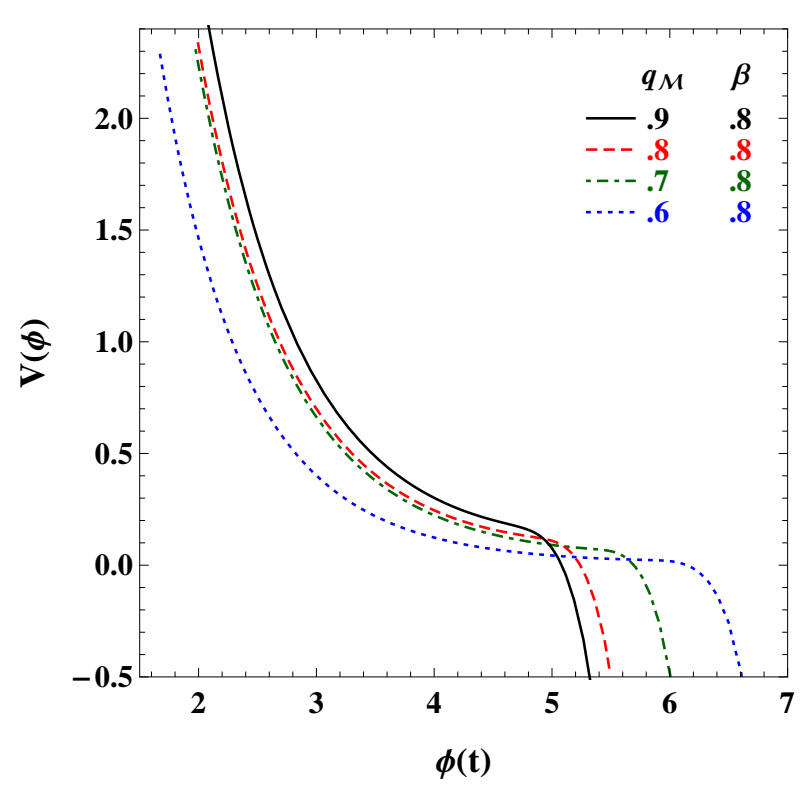

(a)

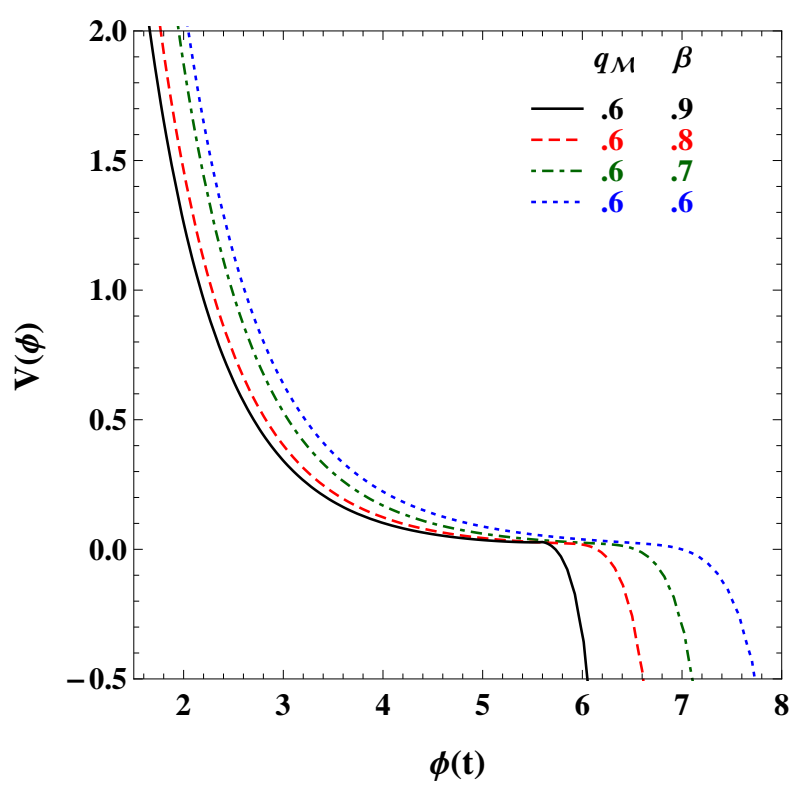

(b)

Figure 4: The left panel (a): potential $V(\phi)$ versus $\phi(t)$ is shown for different values of $q_{\mathcal{M}}$ keeping $\beta$ fixed. The right panel (b): potential $V(\phi)$ versus $\phi(t)$ is shown for different values of $\beta$ keeping $q_{\mathcal{M}}$ fixed. 


\section{Evolution of scalar field in global domain}

Assuming that our global domain is large enough such that it can fit the Friedmannian cosmology, the effective scalar field formulation of the backreaction problem opens up a promising avenue for observational analysis of the effect of backreaction. We now exploit the above correspondence to explicitly reconstruct the scalar field dynamics in a Friedmann universe. For this we consider the quintessence scalar field obeying the equation of motion (3.13, 3.15 ) which mimics the repulsive component in the cosmological evolution. It is noteworthy that the cosmological viability of this scalar field essentially depends on the backreaction parameters in the two domains.

In the flat FRW background, the Hubble parameter can be written as:

$$
H^{2}=\frac{1}{3 M_{p}^{2}}\left(\rho_{m}+\rho_{\phi}\right)
$$

where $\rho_{m}$ and $\rho_{\phi}$ are the energy density of matter and the scalar field, respectively. To investigate the dynamics described by the equation of motion of field (3.15) and the above eqn.(4.1), it would be convenient to cast them as a system of first order equations given by

$$
\begin{gathered}
\frac{d \phi}{d N}=\frac{\dot{\phi}}{h(\phi)} \\
\frac{d \dot{\phi}}{d N}=-3 \dot{\phi}-\frac{1}{h(\phi)} \frac{d V(\phi)}{d \phi}
\end{gathered}
$$

where, $\phi, \dot{\phi}$ and $V$ are dimensionless quantities as defined in Eqn.(3.12) and $N \equiv \ln a$. The function $h$ is given by

$$
h(\phi)=\sqrt{\Omega_{m 0} e^{-3 a}+\frac{\dot{\phi}^{2}}{6}+\frac{V(\phi)}{3}} .
$$

The initial values for $\phi\left(\phi_{i}\right)$ and $\dot{\phi}$ are chosen so as to ensure a viable cosmological model. Since $\phi_{i}$ and $\Omega_{m 0}$ are related by the flatness condition $\left(k=0, \Omega_{m 0}+\Omega_{\phi 0}=1\right)$, an initial value of $\phi_{i}$ can be fixed by it. $\phi_{i}$ controls the deviation from the $\Lambda \mathrm{CDM}$ model. In fig.5 the energy density $\left(\rho_{m}, \rho_{\phi}\right)$ and the density parameter $\left(\Omega_{m}, \Omega_{\phi}\right)$ of matter and the field $\phi$ are plotted. It is seen that during most of the past evolution the energy density of the scalar field is sub-dominant, remaining nearly constant for a long time before becoming dominant around the present time when the energy density of matter falls and becomes comparable to it. We plot equation of state parameter of the scalar field $\omega_{\phi}$ in Fig.6 for different values of $q_{\mu}$ and $\beta$. We notice that in the past the field is frozen at $\omega_{\phi}=-1$, and begins to evolve only around the present time it. It therefore necessarily belongs to the thawing class of scalar field models [33, 34], where at early times, the field gets locked at $\omega_{\phi}=-1$ due to large Hubble damping and waits for the matter energy density to become comparable to the field energy density, which happens at late times. The field then begins to evolve toward larger values of $\omega_{\phi}$ starting from $\omega_{\phi}=-1$. From Fig. 6 we also see that the future acceleration oscillates, and then slows down. This decrease in acceleration essentially depends on the parameter $q_{\mathcal{M}}$. It may be noted that the oscillation in $\omega_{\phi}$ is a feature of the autonomous Eqs.(4.2) and (4.3) considered for the analysis [35]. 


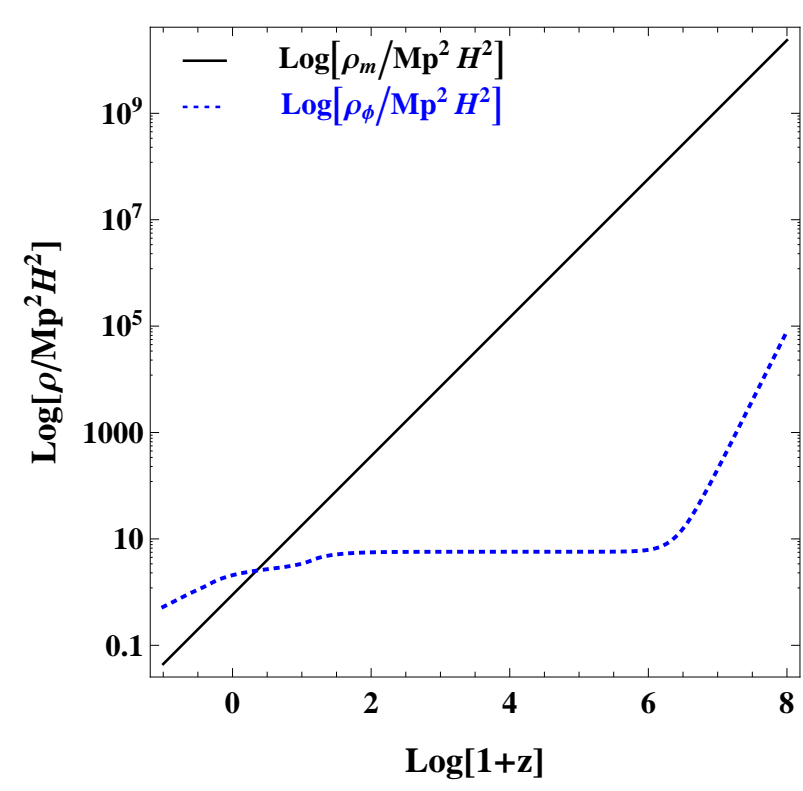

(a)

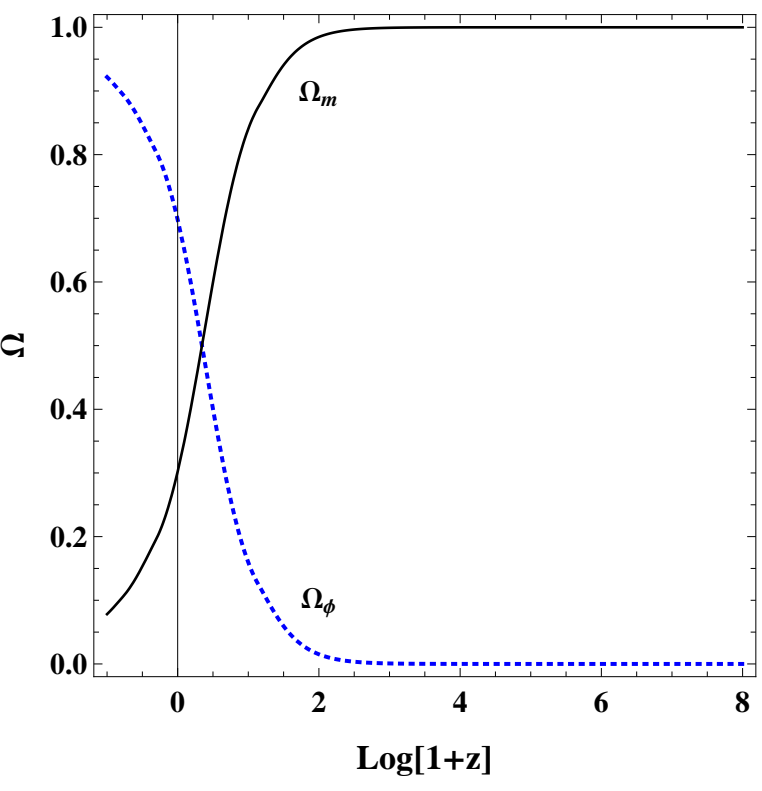

(b)

Figure 5: The left panel (a): Density $\log \left(\frac{\rho}{M_{\mathrm{pl}}^{2} H^{2}}\right)$ for the matter and scalar field are shown for the $q_{\mathcal{M}}=.6$ and $\beta=.9$. The right panel (b): density parameters of $\operatorname{matter}\left(\Omega_{m}\right)$, and field $\left(\Omega_{\phi}\right)$ are shown for the $q_{\mathcal{M}}=.6$ and $\beta=.9$

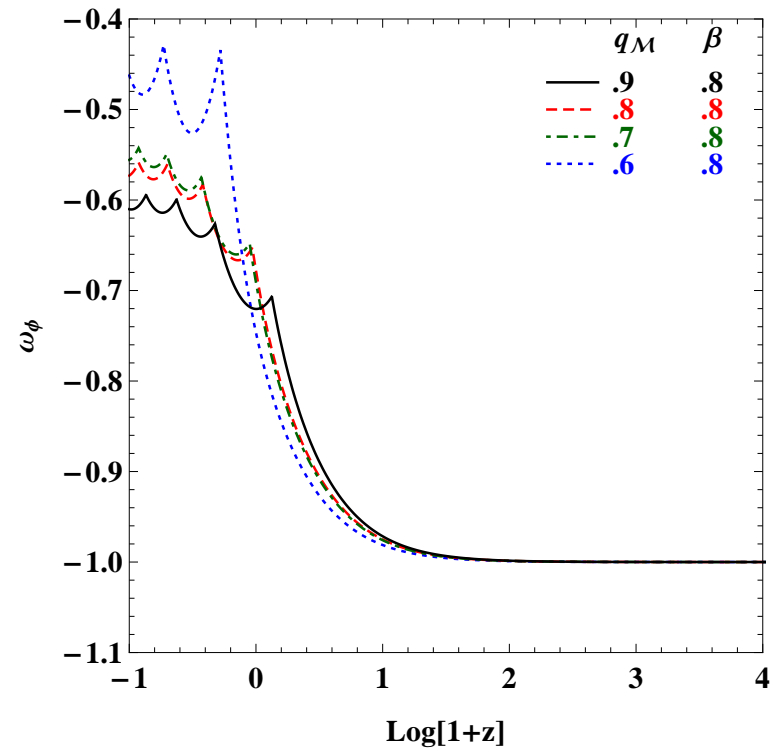

(a)

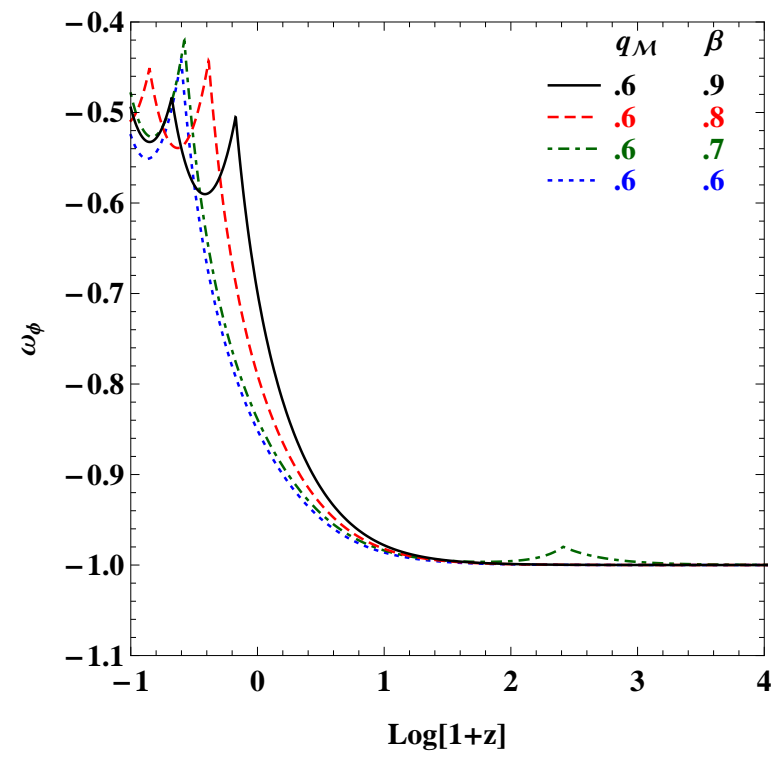

(b)

Figure 6: The left panel (a): the equation of state of the field $\omega_{\phi}$ for various $q_{\mathcal{M}}$ is shown for fixed $\beta$. The right panel (b): $\omega_{\phi}$ for various $\beta$ is shown for fixed $q_{\mathcal{M}}$. 


\section{Data Analysis}

It has been argued that confronting the backreaction models using the cosmological observational data may require more sophisticated approaches [36-39] than what one generally does in case of dark energy models. However, in the present case we have mapped the scalar field originating due to differential scale factors of the local domains into a large global domain such that it can fit the Friedmannian cosmology at the relevant large scales. Therefore, here it is appropriate to use the observational tests where the standard method of reduction of the data assumes the FLRW cosmology.

The evolution of the universe in this set up is controlled by two parameters: one is $\Omega_{m 0}$ the present matter energy density of the universe and the other one is $\phi_{i}$, the initial value of the scalar field. But these two parameters are not completely independent as they can be restrained by imposing the flatness condition on the space part of the universe. In this section, we constrain these two parameters with the assumption of a flat universe by using three types of observational data, viz. the Type 1a Supernovae Union 2.1 compilation [40], the Baryon Acoustic Oscillation (BAO) measurement from the SDSS [41-43], and the Cosmic Microwave Background (CMB) measurement given by WMAP [44].

The Supernovae type Ia (Sn1a) data, UNION 2.1 compilation contains 580 data points, in the form of distance modulus $\mu$ of various Sn1a with the redshift $z$. The distance modulus $\mu$ is defined as

$$
\mu=m-M=5 \log D_{L}+\mu_{0},
$$

where $m$ and $M$ are the apparent and absolute magnitudes of the Supernovae respectively. $D_{L}$ is the luminosity distance defined as

$$
D_{L}(z)=(1+z) \int_{0}^{z} \frac{H_{0} d z^{\prime}}{H\left(z^{\prime}\right)}
$$

and $\mu_{0}=5 \log \left(\frac{H_{0}^{-1}}{M_{p c}}\right)+25$ is a nuisance parameter which should be marginalized. The corresponding $\chi^{2}$ is defined as

$$
\chi_{S N}^{2}\left(\mu_{0}, \theta\right)=\sum_{i=1}^{580} \frac{\left(\mu_{t h}\left(z_{i}, \mu_{0}, \theta\right)-\mu_{o b s}\left(z_{i}\right)\right)^{2}}{\sigma_{\mu}\left(z_{i}\right)^{2}},
$$

where $\mu_{o b s}$ is the observational distance modulus, $\mu_{t h}$ is the theoretical distance modulus for the model, and $\sigma_{\mu}$ is the error in the distance modulus. $\theta$ represents the parameters of the model to be constrained and $\mu_{0}$ is the nuisance parameter. To get rid of $\mu_{0}$, we marginalise it as described in Ref.[45] by defining

$$
\begin{aligned}
& A(\theta)=\sum_{i=1}^{580} \frac{\left(\mu_{t h}\left(z_{i}, \mu_{0}, \theta\right)-\mu_{o b s}\left(z_{i}\right)\right)^{2}}{\sigma_{\mu}\left(z_{i}\right)^{2}}, \\
& B(\theta)=\sum_{i=1}^{580} \frac{\mu_{t h}\left(z_{i}, \mu_{0}, \theta\right)-\mu_{o b s}\left(z_{i}\right)}{\sigma_{\mu}\left(z_{i}\right)^{2}} \\
& C(\theta)=\sum_{i=1}^{580} \frac{1}{\sigma_{\mu}\left(z_{i}\right)^{2}} .
\end{aligned}
$$




\begin{tabular}{|c||c|c|c|c|c|c|}
\hline$z_{B A O}$ & 0.106 & 0.2 & 0.35 & 0.44 & 0.6 & 0.73 \\
\hline \hline$\frac{d_{A}\left(z_{\star}\right)}{D_{V}\left(Z_{B A O}\right)}$ & $30.95 \pm 1.46$ & $17.55 \pm 0.60$ & $10.11 \pm 0.37$ & $8.44 \pm 0.67$ & $6.69 \pm 0.33$ & $5.45 \pm 0.31$ \\
\hline
\end{tabular}

Table 1: Values of $\frac{d_{A}\left(z_{\star}\right)}{D_{V}\left(Z_{B A O}\right)}$ for different values of $z_{B A O}$.

From the above equations one obtains

$$
\chi_{S N}^{2}(\theta)=A-\frac{B^{2}}{C} .
$$

We use the large scale structure data [42] for the BAO co-moving angular-diameter distance ratio $\frac{d_{A}\left(z_{\star}\right)}{D_{V}\left(Z_{B A O}\right)}$, where $z_{\star}(\approx 1091)$ is the decoupling time, $d_{A}$ is given by $d_{A}(z)=$ $\int_{0}^{z} \frac{d z^{\prime}}{H\left(z^{\prime}\right)}$, and $D_{V}(z)=\left(d_{A}(z)^{2} \frac{z}{H(z)}\right)^{\frac{1}{3}}$. Data required for this analysis is compiled in the table 1. We calculate $\chi_{B A O}^{2}$ as described in Ref. [43], by defining

$$
\chi_{B A O}^{2}(\theta)=X_{B A O}^{T}(\theta) C_{B A O}^{-1} X_{B A O}(\theta) .
$$

where,

$$
X_{B A O}(\theta)=\left(\begin{array}{c}
\frac{d_{A}\left(z_{\star}\right)}{D_{V}(0.106)}-30.95 \\
\frac{d_{A}\left(z_{\star}\right)}{D_{V}(0.2)}-17.55 \\
\frac{d_{A}\left(z_{\star}\right)}{D_{V}(0.35)}-10.11 \\
\frac{d_{A}\left(z_{\star}\right)}{D_{V}(0.44)}-8.44 \\
\frac{d_{A}\left(z_{\star}\right)}{D_{V}(0.6)}-6.69 \\
\frac{d_{A}\left(z_{\star}\right)}{D_{V}(0.73)}-5.45
\end{array}\right),
$$

and the inverse covariance matrix given by

$$
C^{-1}=\left(\begin{array}{cccccc}
0.48435 & -0.101383 & -0.164945 & -0.0305703 & -0.097874 & -0.106738 \\
-0.101383 & 3.2882 & -2.45497 & -0.0787898 & -0.252254 & -0.2751 \\
-0.164945 & -2.45499 & 9.55916 & -0.128187 & -0.410404 & -0.447574 \\
-0.0305703 & -0.0787898 & -0.128187 & 2.78728 & -2.75632 & 1.16437 \\
-0.097874 & -0.252254 & -0.410404 & -2.75632 & 14.9245 & -7.32441 \\
-0.106738 & -0.2751 & -0.447574 & 1.16437 & -7.32441 & 14.5022
\end{array}\right)
$$

Lastly, for the constraints from CMB we use the CMB shift parameter

$$
R=H_{0} \sqrt{\Omega_{m 0}} \int_{0}^{1089} \frac{d z^{\prime}}{H\left(z^{\prime}\right)} .
$$

The $\chi_{C M B}^{2}$ is defined as

$$
\chi_{C M B}^{2}(\theta)=\frac{\left(R(\theta)-R_{0}\right)^{2}}{\sigma^{2}},
$$



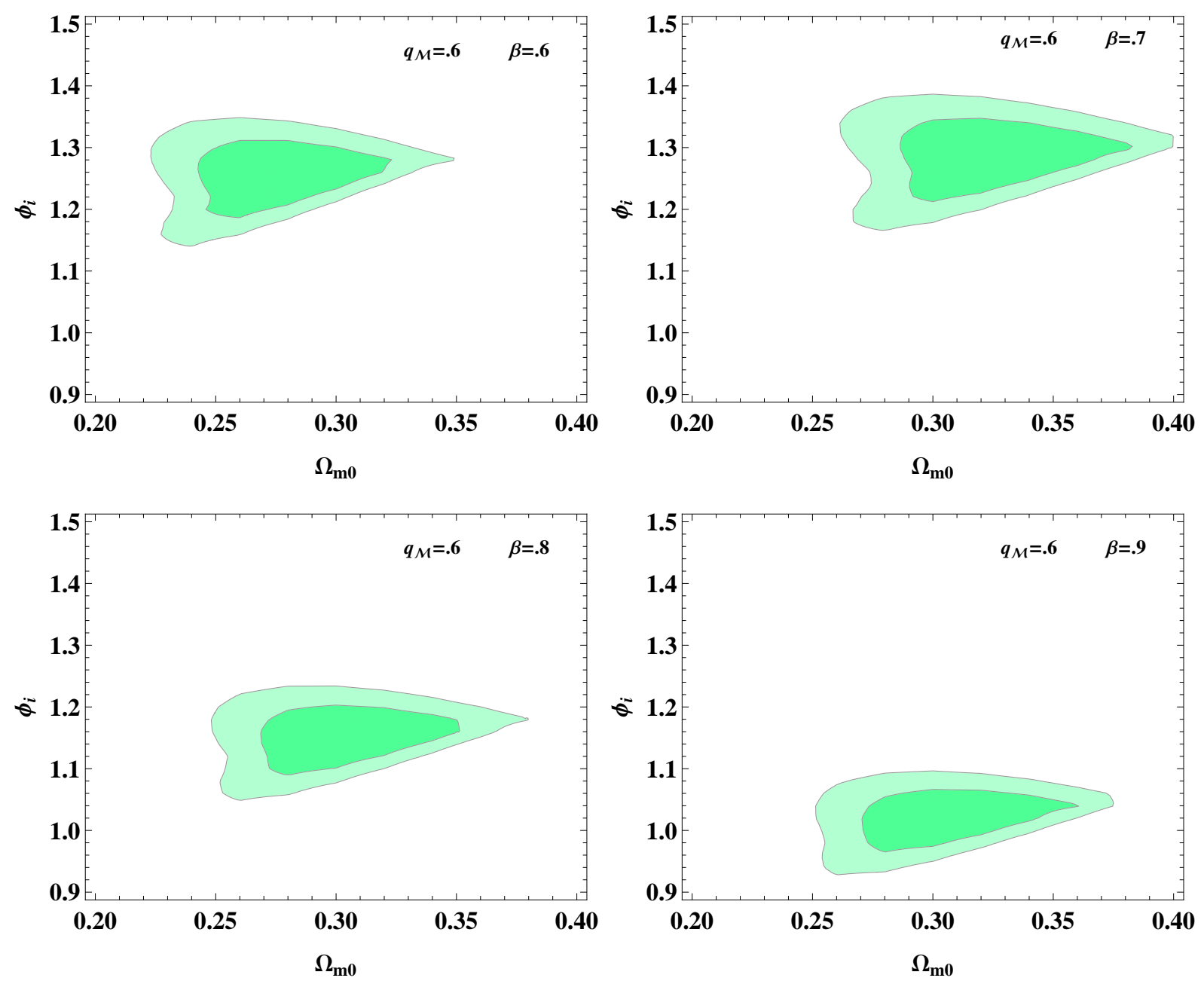

Figure 7: The $1 \sigma$ (dark) and $2 \sigma$ (light) likelihood contours in the $\left(\Omega_{m 0}, \phi_{i}\right)$ phase plane for the total $\chi_{S N+B A O+C M B}^{2}$ for different values of $\beta$.

where, $R_{0}=1.725 \pm 0.018$ [44].

The total $\chi_{\text {total }}^{2}$ combining the three data sets is given by

$$
\chi_{\text {total }}^{2}(\theta)=\chi_{S N}^{2}(\theta)+\chi_{B A O}^{2}(\theta)+\chi_{C M B}^{2}(\theta) .
$$

We carry out this analysis on our model parameters namely, $\phi_{i}$ and $\Omega_{m 0}$. To determine the constraints on $\phi_{i}$ and $\Omega_{m 0}$ we vary $\phi_{i}$ in the range $[0.9,1.5]$ and $\Omega_{m 0}$ in the range $[0.2,0.4]$. Fig.7 shows the $1 \sigma$ and $2 \sigma$ contours in the $\left(\phi_{i}, \Omega_{m 0}\right)$ parameter space for different values of $\beta$ keeping $q_{\mathcal{M}}$ fixed to 0.6. We see that for higher values of $\beta$, the lower values of $\phi_{i}$ and higher values of $\Omega_{m 0}$ is allowed. Fig. 8 shows the contours in parameter space $\left(\phi_{i}, \Omega_{m 0}\right)$, for different values of $q_{\mathcal{M}}$ fixing $\beta=0.8$. We see that for higher values of $q_{\mathcal{M}}$, the parameter space gets more and more constrained, allowing a very small range of initial values of field $\phi_{i}$.

The $\chi^{2}$ statistical measures described above are based on the assumption that the underlying model is a viable one. The analysis is good at finding the best parameters in a model that will fit the data, but is insufficient for deciding whether the model itself is the 

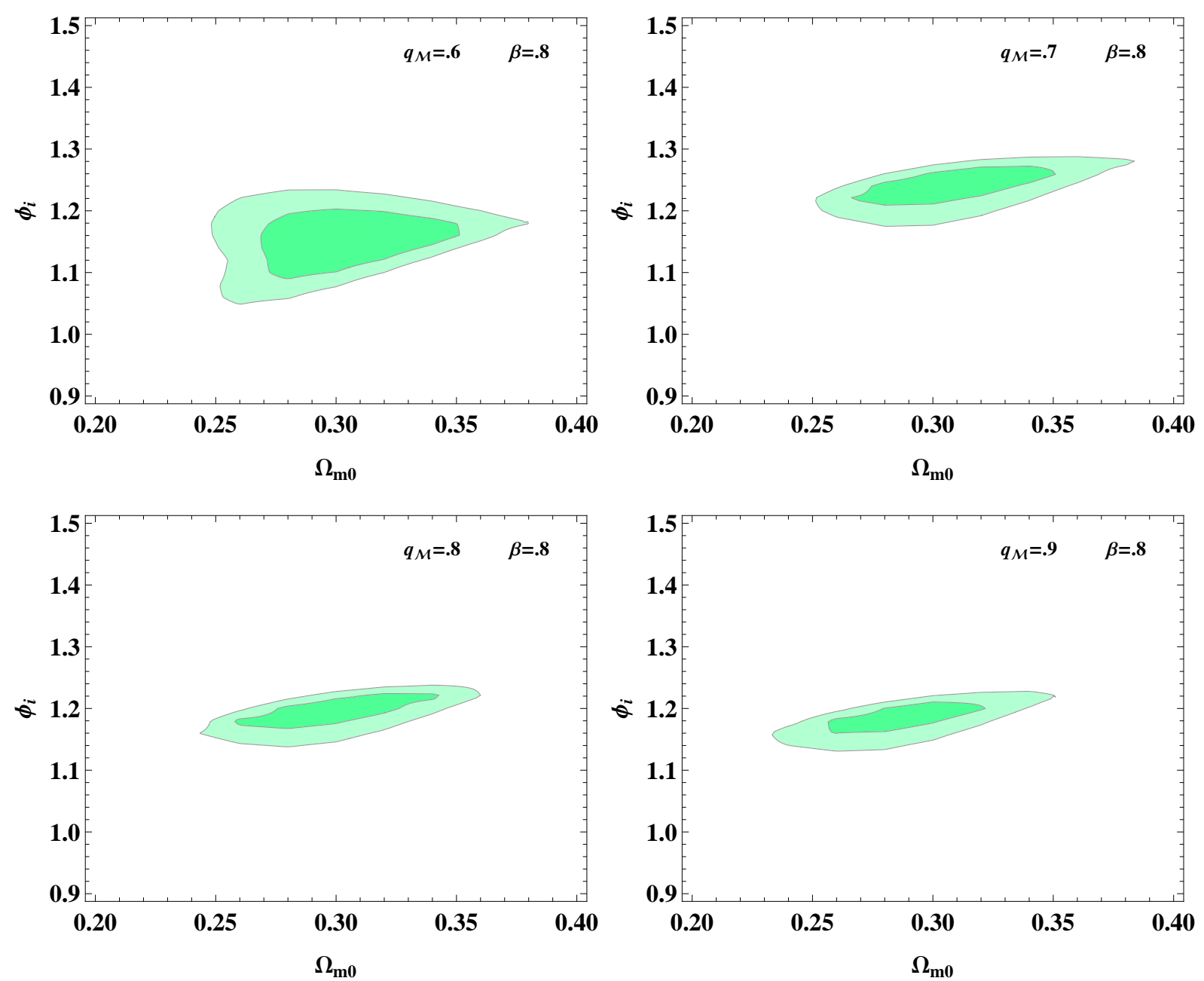

Figure 8: The $1 \sigma$ (dark) and $2 \sigma$ (light) likelihood contours in the $\left(\Omega_{m 0}, \phi_{i}\right)$ phase plane for the total $\chi_{S N+B A O+C M B}^{2}$ for different values of $q_{\mathcal{M}}$.

best one. To assess the strength of the models one uses the information criteria (IC) [46]. The statistics favour models that give a good fit with fewer parameters. We use the Bayesian information criterion (BIC) and Akaike information criterion (AIC) to select the best fit models in comparison to the standard $\Lambda$ CDM model. The AIC and BIC are defined as

$$
\begin{gathered}
A I C=-2 \ln L+2 k, \\
B I C=-2 \ln L+k \ln N,
\end{gathered}
$$

where $L, k$ and $N$ are the maximum likelihood, the number of parameters, and the number of data points, respectively. For Gaussian errors, $\chi^{2}=-2 \ln L$. In order to compare it to the $\Lambda \mathrm{CDM}$ model we find the difference $\triangle \mathrm{AIC}$ and $\Delta \mathrm{BIC}$ from the $\Lambda \mathrm{CDM}$ model of the best fit values of the present model for various $q_{\mathcal{M}}$ and $\beta$ values. In table $2, \Delta \mathrm{AIC}$ and $\Delta \mathrm{BIC}$ are shown, from which we can infer the strength of the model fixed by particular $q_{\mathcal{M}}$ and $\beta$ in comparison to the $\Lambda \mathrm{CDM}$ model. From the table we notice that models for greater $q_{\mathcal{M}}$ and 


\begin{tabular}{|c|c|c|c|}
\hline$q_{\mathcal{M}}$ & $\beta$ & $\Delta \mathrm{AIC}$ & $\Delta \mathrm{BIC}$ \\
\hline \hline .6 & .6 & 8 & 13 \\
\hline .6 & .7 & 9 & 13 \\
\hline .6 & .8 & 10 & 14 \\
\hline .6 & .9 & 10 & 15 \\
\hline .7 & .8 & 24 & 28 \\
\hline .8 & .8 & 26 & 31 \\
\hline .9 & .8 & 28 & 33 \\
\hline
\end{tabular}

Table 2: Values of $\Delta \mathrm{AIC}$ and $\Delta \mathrm{BIC}$ for different values of $q_{\mathcal{M}}$ and $\beta$.

$\beta$ are poorly fitted by the data. For small values of $q_{\mathcal{M}}$ and $\beta$ the model is better fitted than some of the models of dark energy, for instance the Dvali-Gabadadze-Poratti braneworld model [47], considered in Ref. [46].

\section{Conclusion}

In this paper we have employed the Buchert formalism $[18,24,25,27]$ for obtaining the averaged effect of inhomogeneities on the late evolution of the universe. The cosmological evolution is studied here in context of a model constructed using a two-scale void-wall partitioning $[26,30]$. We have performed a detailed analysis of the various aspects of the future evolution of the presently accelerating universe within this framework. The equations governing the dynamics at large global scales in this formalism suggest themselves to interpret the sources of inhomogeneities in geometrical variables in terms of an effective scalar field [31]. We thus choose to consider the model with averaged inhomogeneities at large scales as a variant of the standard FLRW model with matter evolving in a mean field of scalar field $\phi$ representing the backreaction terms. The potential of this scalar field captures the geometrical signatures of structures in universe and is free from phenomenological parametrisation. The investigation of the field dynamics in the global domain, where the the cosmological principle is intact is done in details. It is seen that the field resembling a thawing dark energy model gives rise to a transient acceleration at present.

We have imposed observational constraints on the model parameters $\phi_{i}$ and $\Omega_{m 0}$ using the data from SNIa [40], BAO [41-43], and CMB [44] observations. By constructing the corresponding contour plots we deduce that $\phi_{i}$ is constrained by the data to a small range of values, depending upon the values of individual expansion rates of the two sub-domains, i.e., the void and the wall. The present density parameter of matter $\Omega_{m 0}$ is constrained around the concordance values. We have also checked the strength of the model in comparison with the $\Lambda$ CDM model using Bayesian information criterion (BIC) and Akaike information criterion (AIC) [46]. Though the present toy model is not favoured by the data, it still enables a better fit compared to some other proposed models of dark energy involving modifications [47] of standard GR. The results obtained by us in the present work follow from the choice of our ansatz regarding the wall and void scale factors which reflect, at best, the qualitative expectations of the physical behaviour of overdense and underdense regions, respectively. Further work remains to be done where more realistic and observationally compatible properties of the void and wall regions could be incorporated within the formalism. Further investigations in context of more realistic models involving multidomain scenarios is called for, especially 
in view of a remarkable feature of our results showing that the averaged effect of inhomogeneities render the current global acceleration to a transient phenomenon, which dies down in future.

\section{ACKNOWLEDGEMENTS}

The authors thank Thomas Buchert and Nilok Bose for useful discussions.

\section{References}

[1] S. Perlmutter et al, Astrophys. J. 517 (1999) 565,

A. G. Riess et al Astron. J. 116 (1998) 1009.

[2] D. N. Spergel et al (WMAP Collaboration), Astrophys. J. Suppl. Ser. 170 (2007) 377.

[3] U. Seljak et al (SDSS Collaboration), Phys. Rev. D 71 (2005) 103515.

[4] D. J. Eisenstein et al (SDSS Collaboration), Astrophys. J. 633 (2005) 560.

[5] B. Jain and A. Taylor, Phys. Rev. Lett. 91 (2003) 141302.

[6] S. Weinberg, Rev. Mod. Phys. 61 (1989) 1, T. Padmanabhan and H. Padmanabhan, Int. J. Mod. Phys. D 23 (2014) 1430011.

[7] P. J. E. Peebles and A. Vilenkin, Phys. Rev. D 59 (1999) 063505, A. S. Majumdar, Phys. Rev. D 64 (2001) 083503.

[8] V. Sahni, Lect. Notes Phys. 653 (2004) 141.

[9] E.J. Copeland, M. Sami and S. Tsujikawa, Int. J. Mod. Phys. D 15 (2006) 1753.

[10] N. Bose and A. S. Majumdar, Phys. Rev. D 79 (2009)103517, N. Bose and A. S. Majumdar, Phys. Rev. D 80 (2009) 103508.

[11] A Joyce, B Jain, J. Khoury and M. Trodden, Physics Reports 568 (2015) 1.

[12] Amna Ali, Radouane Gannouji, M. Sami and Anjan A.Sen; Phy. Rev. D 81 (2010) 104029.

[13] Amna Ali, Radouane Gannouji and M. Sami, Phys.Rev.D 82 (2010) 103015.

[14] Amna Ali, Radouane Gannouji, Md. Wali Hossain and M. Sami, Phys.Lett. B 718 (2012) 5-14.

[15] Debabrata Adak, Amna Ali and Debasish Majumdar, Phys. Rev. D 88 (2013) 024007.

[16] R.M. Zalaletdinov, Gen. Rel. Grav. 24 (1992) 1015, R.M. Zalaletdinov, Gen. Rel. Grav. 25 (1993) 673.

[17] A.A. Coley, N. Pelavas and R.M. Zalaletdinov, Phys.Rev.Lett. 95 (2005) 151102.

[18] T. Buchert, Gen. Rel. Grav. 32 (2000) 105,

T. Buchert, Gen. Rel. Grav. 33 (2001) 1381.

[19] M. Korzyński, Class. Quantum Grav. 27 (2010) 105015.

[20] T. Clifton, K. Rosquist, and R. Tavakol; Phys. Rev. D 86 (2012) 043506.

[21] H. Skarke, Phys. Rev. D 89 (2014) 043506.

[22] T. Buchert et al, Class. Quantum Grav. 32 (2015) 215021.

[23] T. Buchert et al, Int. J. Mod. Phys. D 25 (2016) 1630007.

[24] T. Buchert and M. Carfora, Phys. Rev. Lett. 90 (2003) 031101.

[25] T. Buchert, M. Carfora, Class. Quant. Grav. 25 (2008) 195001.

[26] A. Wiegand, T. Buchert; Phys. Rev. D 82 (2010) 023523. 
[27] T. Buchert and S. Rasanen, Annu. Rev. Nucl. Part. Sci. 62 (2012) 57.

[28] S. Rasanen and J. Cosmol, Astropart. Phys. 0402 (2004) 003, S. Rasanen and J. Cosmol, Astropart. Phys. 0804 (2008) 026, S. Rasanen and J. Cosmol; Astropart. Phys. 02 (2009) 011, S. Rasanen, Phys. Rev. D 81 (2010) 103512.

[29] D. L. Wiltshire, New J. Phys. 9 (2007) 377,

D. L. Wiltshire, Phys. Rev. Lett. 99 (2007) 251101.

[30] N. Bose and A. S. Majumdar, MNRAS Letters 418 (2011), L45, N. Bose and A. S. Majumda, Gen. Rel. Grav. 45 (2013) 1971.

[31] Thomas Buchert et al, Class. Quant. Grav. 23 (2006) 6379-6408.

[32] Rodrigo M. Barbosa et al, Gen. Relativ. Gravit. 48 (2016) 51.

[33] R. R. Caldwell, Eric V. Linder, Phys. Rev. Lett. 95 (2005) 141301.

[34] Amna Ali, M. Sami and A. A. Sen, Phys. Rev. D 79 (2009) 123501.

[35] M. Sami and T. Padmanabhan, Phys. Rev. D 67 (2003) 083509.

[36] D.L. Wiltshire, Phys. Rev. D 80 (2009) 123512.

[37] P.R. Smale and D.L. Wiltshire, Mon. Not. R. Astr. Soc. 413 (2011) 367.

[38] J.A.G.Duley, M.A. Nazer and D.L. Wiltshire, Class. Quantum Grav. 30 (2013) 175006.

[39] M.A Nazer and D.L. Wiltshire, Phys. Rev. D 91 (2015) 063519.

[40] N. Suzuki et al, Astrophys. J. 746 (2012) 85.

[41] D. J. Eisenstein et al, Astrophys. J. 633 (2005) 560.

[42] W. J. Percival et al, Mon. Not. Roy. Astron. Soc. 381,(2007) 1053, W. J. Percival et al, Mon. Not. Roy. Astron. Soc. 401, 2148 (2010), F. Beutler et al, Mon. Not. R. Astron. Soc. 416, 3017 (2011), N. Jarosik et al, Astrophys. J. Suppl. Ser. 192, 14 (2011).

[43] C. Blake et al, Mon. Not. R. Astron. Soc. 418 (2011) 1707.

[44] E. Komatsu et al, Astrophys. J. Suppl. Ser. 192 (2011) 18.

[45] R. Lazkoz, S. Nesseris and L. Perivolaropoulos, JCAP 0511 (2005) 010.

[46] T. M. Davis et al, Astrophys. J. 666 (2007) 716.

[47] G. Dvali, G. Gabadadze and M. Poratti, Phys. Lett. B 484 (2000) 112. 